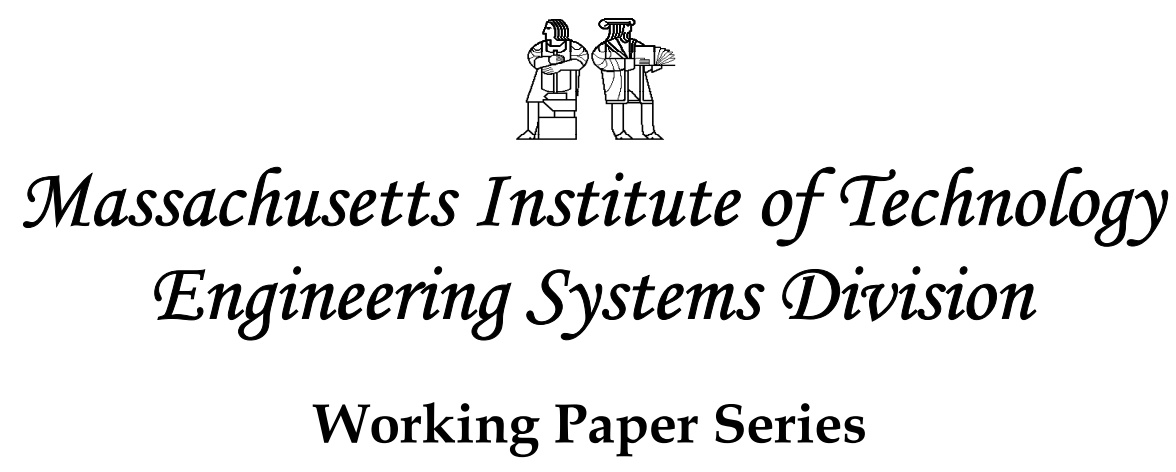

ESD-WP-2001-04

\title{
EXTRACTING THE ESSENCE OF FLEXIBILITY IN SYSTEM DESIGN
}

\author{
JOSEPH H. SALEH \\ GRADUATE RESEARCH AsSISTANT \\ DANIEL E. HASTINGS \\ Professor of AERONAUTICS AND AstronaUtics \\ AND ENGINEERING SYSTEMS \\ DAVA J. NEWMAN \\ Associate Professor of Aeronautics AND Astronautics \\ DePARTMENT OF AERONAUTICS AND ASTRONAUTICS \\ MASSACHUSETTS INSTITUTE OF TECHNOLOGY
}




\section{Extracting the Essence of Flexibility in System Design}

\author{
Joseph H. Saleh \\ Graduate student \\ jsaleh@mit.edu
}

\author{
Daniel E. Hastings \\ Professor of Aeronautics and Astronautics \\ and Engineering Systems, AIAA Fellow
}

\author{
Dava J. Newman \\ Associate Professor of Aeronautics and \\ Astronautics, and MacVicar Faculty \\ Fellow, AIAA Senior Member
}

Department of Aeronautics and Astronautics, Massachusetts Institute of Technology, Cambridge, MA 02139

\begin{abstract}
The modest purpose of this paper is to review the concept of flexibility as discussed in various fields of investigations, and to extract its characteristic features. In order to discuss any subject matter clearly, it is necessary to begin with a clear set of definitions. Indeed much can be gained through careful and consistent definitions of terms alone. Flexibility however is a word rich with ambiguity. While it is being increasingly used in various fields, few attempts have been made to formally define, quantify, and propose ways for achieving flexibility. This paper proposes to fill in part this gap by synthesizing a clear and consistent definition of flexibility. It will do so by reviewing the usage of the term in various fields of inquiries, and show that it is indeed possible to clearly and unambiguously characterize flexibility, and to disentangle it from closely related concepts.
\end{abstract}

\section{Flexibility: A Word Rich with Ambiguity}

Flexibility has become in recent years a key concept in many fields, particularly in most design endeavors. Indeed, for a multitude of disciplines, such as urban planning [1], architecture [2], finance [3], manufacturing [4], software design [5] and others, flexibility is hailed as critical. However, few attempts have been made to formally and unambiguously define it. Intuitively, flexibility is understood as the ability to respond to change. Although essential, this feature nevertheless fails to distinguish it from other properties such as robustness. Furthermore, the literature on design is replete with terms related to a system's ability to handle change, such as adaptability, changeability, agility, elasticity, etc. But when one seeks to grasp their concrete content, such terms often fail.

One source of ambiguity therefore arises from the failure of the familiar characterization of flexibility, i.e., the ability to handle change, to distinguish it from other properties, particularly in the light of the proliferation of its pseudo-synonyms.
The following extract [6] is a good representative of this ambiguity where flexibility and robustness are used almost interchangeably, and in which "robust design [provides] flexible solutions":

"The robust design concept is extended to make decisions that are flexible to be allowed to vary within a range (called type II robust design)...The concept behind type II robust design for providing flexible solutions is represented below. For purposes of the illustration, assume that the performance is a function of only one variable $x$. Generally, in this type of robust design, to reduce the variation of response caused by variations of the design variables, instead of seeking the optimum value, a designer is interested in identifying the flat part of a curve near the performance target. If the objective is to move the performance towards $\mathrm{M}$ and if a robust design is not sought, then obviously $x=\mu_{\text {opt }}$ is a better choice. However for a robust design $x=\mu_{\text {robust }}$ is a better choice."

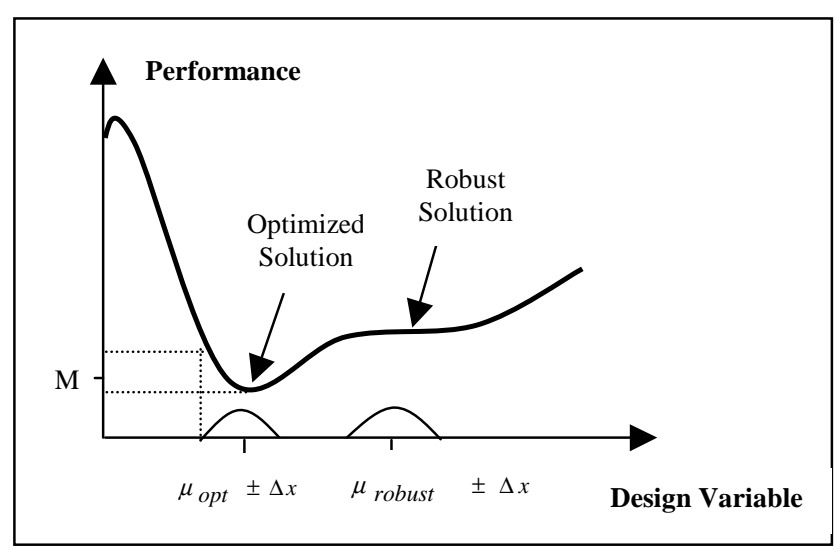

Figure 1. Type II robust design: developing flexible solutions. Adapted from [6]. Variation of the design parameter around $\mu_{\text {opt }}$ causes greater variation in the performance than when the design parameter is set to $\mu_{\text {robust }}$. 
The above example sets the objective of "achieving flexible solutions" and equates that with (type II) robust solutions. Flexibility is thus turned into a by-product of the robust design methodology. It is arguable however whether such a discussion captures any distinctive feature of flexibility.

A comprehensive treatment of flexibility in system design should address the following questions:

1. What is flexibility?

2. Why or when is flexibility needed in system design?

3. How can we design for flexibility? What are the design principles for embedding flexibility in system design?

4. What are the trade-offs associated with designing for flexibility? What is the value of flexibility and what are the penalties (cost, performance, risk, etc.), if any, associated with it?

The literature of the different fields of inquiries mentioned above seldom addresses these questions holistically. Instead the focus is on one particular question at the detriment of the others, often the first and second question. The literature on Real Options is good example of this trend where the focus is primarily on capturing the value of flexibility. The following extract [7] is the opening paragraph of a reference text on the subject; it illustrates the emphasis of the subject on the value of flexibility:

"Flexibility has value. While this statement is obvious at the conceptual level, it is surprisingly subtle at the applied level. Professional managers have long intuited that [flexibility is an important element] in valuation and planning decisions. But precisely how valuable is flexibility and how can its value be quantified?"

This paper will focus on the first question: It proposes to review the concept of flexibility as discussed in different fields of investigations, and to extract its characteristic features. The objective is to synthesize a clear and consistent definition of flexibility, and to disentangle it from its pseudo-synonyms and related properties. The following questions will be addressed separately in the subsequent papers.

The paper is organized as follows: Section 2 provides a selected literature review of different fields of investigations that have addressed issues of flexibility. Section 3 proposes a definition of flexibility of a design, and carefully disentangles it from discussions on flexibility in the design process. A brief literature review of Robust Control and Robust Design is also provided and a definition of robustness is synthesized. Flexibility (of a design) and robustness are then contrasted, and a distinction is drawn as well between flexibility and universality of a design. Section 4 discusses three examples of flexible systems and the need for flexibility in system design, and illustrates the relationship between flexibility and a system's design lifetime. Section 5 touches on issues of flexibility in the context of distributed satellite systems. Section 6 contains the summary and conclusions.

\section{Discussions of Flexibility: A Selected Literature Review}

This section briefly reviews the various definitions of flexibility provided by three distinct fields of investigations: Flexibility in manufacturing systems, flexibility in multidisciplinary design processes, and real options thinking and managerial flexibility.

\subsection{Flexibility in Manufacturing Systems}

In the manufacturing community, different types of flexibility are defined based on the nature of change the production system can accommodate. The sheer amount of literature on Flexible Manufacturing Systems (FMS) is daunting. A great number of topics are addressed ranging from the design of manufacturing cells and machine grouping, to the scheduling, loading, and control of FMS [4]. This section briefly reviews a handful of definitions among the numerous types of flexibility that are defined in this literature. Volume flexibility is defined as the ability of a production system to handle changes in daily or weekly volume of the same product, thus allowing the factory to operate profitably at varying overall production levels. Product mix flexibility is defined as the ability to manufacture a variety of products without major modification of existing facilities. Routing flexibility is defined as the ability to process a given set of parts on alternative machines. Operation flexibility is defined as the ability to interchange the ordering of operations on a given part, thus allowing the ease of scheduling of its production $[8,9]$.

Flexibility in this environment is not only viewed as a reactive capability, it is also regarded as a competitive weapon which not only allows a company to respond to change, but also to create change and set the market pace for rapid production and innovation [10].

Agility is another term related to the ability to respond to change. It was first introduced in manufacturing environments then broadened to encompass the extended enterprise. It is often loosely defined, and used to characterize different things in a business environment. For instance, in Pathways to Agility, Oleson (1998) describes "agile strategic planning processes", "agile 
automation", and discusses the need for "agile business relationships" with suppliers and customers. He defines agility as the "ability to respond with ease to unexpected but anticipated events" ". Similarly, Fricke et al. (2000) define agility as the "property of a system to implement changes rapidly", and flexibility as the "property of a system to be changed easily and without undesired effects." "Agility" is thus used as a desired qualitative attribute for an enterprise to thrive in a hyper-competitive environment. It is difficult however to see how the definitions of flexibility and agility provided by Fricke et al. (2000) differ or overlap, and to grasp the concrete content of "agility".

\subsection{Multidisciplinary Design and Flexibility in the Design Process}

Current research has addressed the issue of flexibility in multidisciplinary design ${ }^{2}$. The focus of those efforts has been on achieving "flexibility in the design process." Typical approaches have consisted of incorporating designers' preferences with degrees of satisfaction in specifying design requirements. Thurston (1991) for example uses utility theory based preference functions to express designers' preference over single or multiple attributes. Wallace et al. (1996) define specification functions to indicate the subjective probability that performance levels are achieved. Mohandas and Sandgren (1989) recommend the use of fuzzy goals to model the degree of satisfaction level.

These approaches, along with others such as the interval methods and probabilistic-based methods, were developed, according to Chen and Yuan (1997), in response to the following concern: "How does one capture the uncertainty-which characterizes the early stages of design-and offers flexibility in specifying the design requirements so that the designs that are marginally outside the precise level of performance are not worthless?"

Chen and Lewis (1999) define their understanding of flexibility in the design process as follows:

"Our aim is to provide flexibility in the design process and to help further resolve the conflicts and disputes of rationality between the interests of multiple disciplines. By flexibility we mean that instead of looking for a single point solution in one discipline's model, we look for a range of solutions that involve information passing between multiple players (disciplines). With this

\footnotetext{
${ }^{1}$ [Oleson, 98], pp. xvi.

2 Multiple technical disciplines involved in a common design endeavor. Expression used to reflect the interdisciplinary nature of complex systems design [6].
}

flexibility, the design freedom of individual disciplines [...] could be significantly improved. Ultimately, this process will result in better products in less time because fewer iterations are needed."

Flexibility in the design process therefore entails expressing degrees of desirability in specifying design requirements. A recent report by the United States General Accounting Office on Best Practices in requirements specifications [11] echoes this description and emphasizes the need for a flexible behavior on behalf of the customers and developers in setting requirements:

"Flexibility in setting requirements is key to closing gaps between customer expectations and developer resources. While knowledge is essential to identifying gaps between expectations and resources, it takes flexibility on part of both the customer and the product developer to close the gaps. Flexibility represents the customer's ability and willingness to lower product expectations, coupled with the product developer's willingness and ability to invest more resources to reduce technical risks and other gaps before program start [...] In successful cases, requirements were flexible until the decision was made to commit to product development [...] This made it acceptable to reduce, eliminate, or defer some customer wants so that the product's requirements could be matched with the resources available to deliver the product within the desired cycle time."

\subsection{Real Options and Managerial Flexibility}

Today's market require that important investment decisions be made in very uncertain environments, when the market size, the time to market, the cost of development, the competitors' moves, and so on simply are not known.

Managerial flexibility ${ }^{3}$ refers to the ability of management to affect the course of a project by acting in response to the resolution of market uncertainty over time. A flexible project may allow for downside protection against unfavorable market events, e.g., by abandoning the project, or introduce growth opportunities in the case of favorable conditions. Thus managerial flexibility reduces a project's exposure to uncertainty while providing management with the ability to respond to unfolding events. This concept is introduced in the context of Decision Tree Analysis and Real Options thinking. It is used in making a persuasive case against traditional valuation tools for capturing the value of staged or contingent investments (option to initiate a

\footnotetext{
${ }^{3}$ The expression was first introduced by Trigeorgis and Mason in "Valuing Managerial Flexibility" in Midland Corporate Finance Journal, 5-1987. pp. 14-21.
} 
project, option to expand, to wait-and-see, etc.). A growing body of literature exists that describes the shortcoming of Discounted Cash Flow tools such as the classical NPV or IRR, and proposes ways of applying "Option Thinking" to valuing managerial flexibility. The reader is referred to Trigeorgis and Mason (1987), Triantis (1990), Faulkner (1996), or Amram and Kulatilaka (1999) for more elaborate discussions of option thinking and managerial flexibility.

On a parallel note, a plan of action is called rigid if it contains few contingent decisions, and flexible if it contains many such decisions. Plans made long in advance of the "action" are normally associated with rigidity, thus implying that to be flexible, one must be willing to wait and see, to defer decisions until one has taken into account the way a situation develops. Hence flexibility in this context implies remaining uncommitted to the extent of allowing oneself some leeway to design ways of dealing with unforeseen events [12].

\section{Flexibility of a Design}

A common theme across the previous discussions of flexibility is the ability to handle change. This characterization of flexibility however is not sufficient to distinguish it from other properties such as robustness. The ambiguity arises from the ill-defined term "change". A clear definition of flexibility should provide the following information:

- A time reference associated with the occurrence of change, i.e., when is the "change" happening during the life cycle of the system.

- A characterization of what is changing, e.g., the system's environment, the system itself, or the customer's needs of the system.

- An indication for providing metrics of flexibility, or the ability to rank different designs according to their flexibility.

\subsection{Time Frame Attached to a System's Life Cycle}

A system's life cycle starts with the identification of customer's needs and proceeds towards the definition, design, production, operations, and disposal of a particular system. Prior to fielding, the process needn't be sequential: Different development models exist, e.g., the waterfall model, the spiral model, that offer a particular perspective, insights, and solutions to product development lifecycle problems. Each model generally constraints the sequence in which work is performed starting when the product is conceptualized and ending when the product has satisfied the acceptance criteria [13].

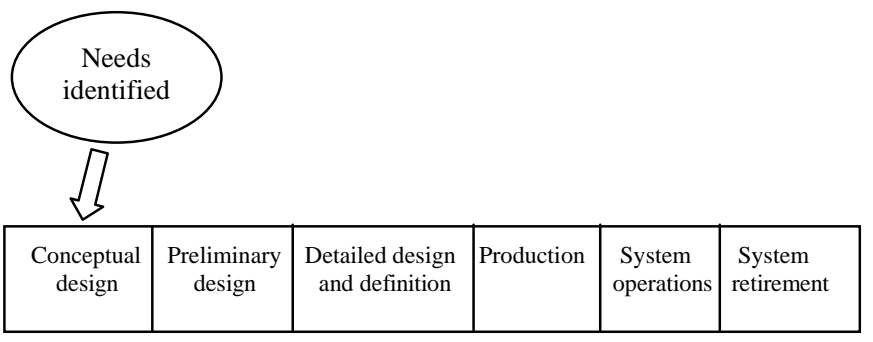

Figure 2. Example of a system life cycle. Adapted from [14]

In the particular case of a space system, the life cycle typically progresses through four phases [15]:

- Concept exploration, the initial study phase of a space mission which results in a broad definition of the space mission and its components.

- Detailed development, the formal design phase, which results in a detailed definition of the system components and, in larger programs, development of test hardware or software.

- Production and deployment, the construction of the ground and flight hardware and software and launch of the full constellation of satellites.

- Operations and support, the day-to-day operation of the space system, its maintenance and support, and finally its de-orbit or recovery at the end of the mission life.

These phases are named differently depending on whether the sponsor is NASA or DoD or some other agency.

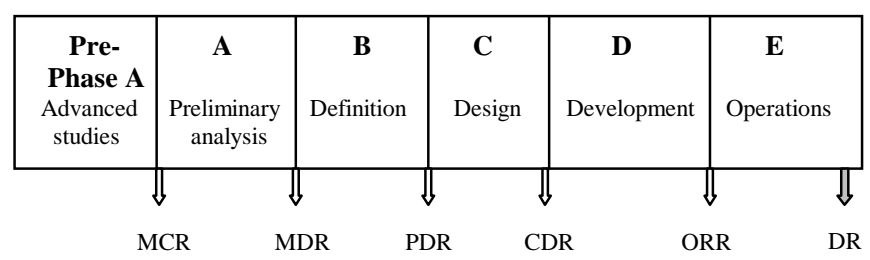

Figure 3. Above, NASA's space program development phases and the associated "gates" or milestones of the program: MCR mission concept review, MDR mission design review, PDR preliminary design review, CDR critical design review, ORR operational readiness review, DR decommissioning review. Not all the program's milestones are represented. Adapted from [15].

The system's life cycle provides an appropriate time reference for our purposes as described below: 


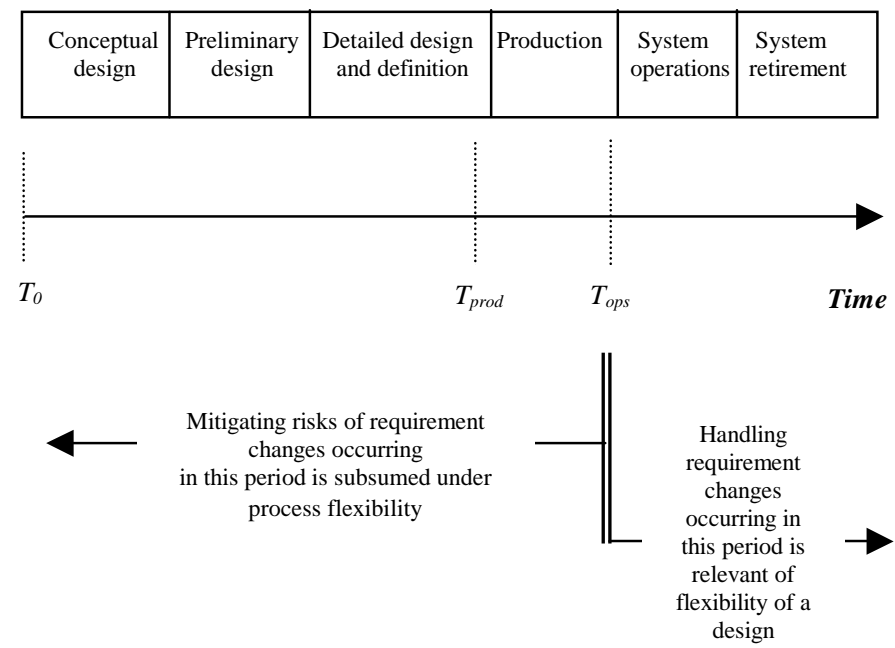

Figure 4. Time frame attached to a system's life cycle, and time periods associated with process flexibility versus flexibility of a design.

Although the development process is rarely sequential, the program's milestones, e.g., the preliminary design review (PDR) and the critical design review (CDR), ensure that $T_{p r o d}$ and $T_{\text {ops }}$ are well defined in the case of space systems ${ }^{4}$. Changes may occur any time.

Current research that have addressed the issue of achieving flexibility in the multidisciplinary design $[6,16,17]$ have dealt with different ways of specifying requirements and handling their dynamics or changes occurring prior to $T_{\text {ops }}$. This was undertaken in order to resolve the conflicts of rationality between the interests of multiple disciplines involved in a common design endeavor. This is the time period-prior to $T_{o p s}$-with which "flexibility in the design process" is concerned. Process flexibility include activities, methods and tools devised to mitigate the risks-cost, schedule, and performance-resulting from requirement changes occurring before fielding a system (see Fig. 4).

This is not the focus of this work. We will mainly be concerned with changes occurring after $T_{o p s}$. But what are these changes about? Changes can occur in the system's environment (political, cultural, organizational, physical, etc.), in the system itself (e.g., wear and tear), or in its requirements-capabilities and attributes-resulting from changing customer needs.

\footnotetext{
${ }^{4}$ In the case of other artifacts, $T_{\text {ops }}$ is always well defined irrespective of the development model, e.g., waterfall, or spiral model.
}

\subsection{Definition: Flexibility of a Design}

We define flexibility of a design as the property of a system that allows it to respond to changes in its initial objectives and requirements - both in terms of capabilities and attributes-occurring after the system has been fielded, i.e., is in operation, in a timely and cost-effective way.

"Requirements", "capabilities" and "attributes" are used in the sense defined by the IEEE Standard 1233, 1998 Edition:

A requirement is:

(a) A condition or capability needed by a user to solve a problem or achieve an objective.

(b) A condition or capability that must be met or possessed by a system or system component to satisfy a contract, standard, specification, or other formally imposed document.

(c) A documented representation of a condition or capability as in definition of (a) or (b).

Requirements can be taken from customer needs and can be derived from technical analysis.

Capability (or functionality): Capabilities are the fundamental requirements of the system and represent the features or functions of the system needed or desired by the customer. A capability should usually be stated in such a way that it describes what the system must do. The capability should be stated in a way that is solution independent.

This definition provides a mean for distinguishing between requirements as capabilities and the attributes of these requirements. The following examples illustrate this distinction:

1. Requirement: Move people from New York to California at a maximum speed of $5300 \mathrm{~km} / \mathrm{hr}$ [18].

Capability: Move people between California and New York

Attribute: Cruising speed of $2500 \mathrm{~km} / \mathrm{hr}$

Constraint: Maximum speed of $5300 \mathrm{~km} / \mathrm{hr}$

2. Requirement: The Mars Global Surveyor Spacecraft shall be capable of providing delta-V of $1290 \mathrm{~m} / \mathrm{s}$, inclusive of finite burn losses from thrust vector misalignment, gravity losses, and all other maneuver inefficiencies [19]. 
Capability: Provide propulsive capability

Attribute: Delta-V $=1290 \mathrm{~m} / \mathrm{s}$

Constraints: Despite various losses (thrust

vector misalignment, gravity, etc.)

A corollary of our definition of flexibility is that a flexible system can be modified in a timely and costeffective way in order to satisfy different requirements at different points in time. These requirements, or requirement changes, as well as the time of occurrences of these changes, can be known or unknown a priori.

Examples of flexible designs will be discussed shortly after the concept of flexibility is disentangled from that of robustness and universality.

\subsection{On Robustness: A Brief Survey of Robust Control and Robust Design}

As stated previously, the distinction between the two concepts, robustness and flexibility, is a subject rich with ambiguity. Any attempt to define flexibility should address this issue. In order to discuss this concern, the following paragraphs review the concept of robustness as devised in two major areas of engineering undertaking, namely in feedback control systems-Robust Controland Robust Design, also known as Taguchi's method. The purpose of this discussion is present a conceptual understanding of robustness of a design so that it forms a background against which the above definition of flexibility can be contrasted.

3.3.1. Robust Control. Controls engineers have developed a set of sophisticated mathematical tools to handle disturbances and model uncertainty in systems they wish to control. The main ingredients of present day robust control theory were already present in the classical work of Bode in 1945 [36]. The following discussion addresses some of the key ideas underlying Robust Control. The reader interested in the subject can review the work by Francis (1987), Doyle et al. (1992), Ackermann (1993), Dahleh et al. (1995), or R. SanchezPena et al. (1998).

The goal of Robust Control and the essence of robustness-from a system's control perspective-are clearly stated by Stefani et al. (1994):

"The ultimate goal of a control-system designer is to build a system that will work in the real environment. Since the real environment may change with timecomponents may age or their parameters may vary with temperature or other environmental conditions-or the operating conditions may vary-load change, disturbances-the control system must be able to withstand these variations.

Assuming the environment does not change, the second fact of life is the issue of model uncertainty. A mathematical representation of a system often involves simplifying and sometimes wishful assumptions. Nonlinearities are either unknown, and hence unmodeled, or modeled and later ignored to simplify the analysis. Different components of systems-actuators, sensors, amplifiers, gears, belts-are sometimes modeled by constant gains, even though they may have dynamics and nonlinearities. Dynamic structures, e.g., aircrafts, satellites, missiles, have complicated dynamics in high frequencies, and these may initially be ignored. Since control systems are typically designed using muchsimplified models of systems, they may not work on the real plant in real environments.

The particular property that a control system must possess in order for it to operate properly [ensure stability and achieve a set of pre-defined performance specifications] in realistic situations is called robustness."

The above are some of the key conceptual issues Robust Control deals with. Next we examine some of the fundamentals of the Robust Design methodology. While these two fields-Robust Control and Robust Designhave rarely interacted, they have nevertheless manipulated similar concepts and dealt with comparable problems at some level of abstraction, even though their tools and their specific domain of applicability differ. The purpose of the following discussion is to extract the essence of robustness in the particular field of Robust Design.

3.3.2. Robust Design. Robust Design is a design methodology developed in order to make a product's performance insensitive to raw material variation, manufacturing variability, and variations in the operating environment [20]. It was developed in the late 1950s by Genishi Tagushi [21] and builds upon ideas from statistical experimental design.

Robust products work well even when produced in real factories and used by real customers under real conditions of use. For instance when buying a car, a customer wants one that will start readily in northern Canada in the winter and not overheat in southern Arizona in the summer for example. In other words, he or she wants a car that is robust with respect to variations of use conditions. He or she also prefers a car that is as good at 50,000 miles as when new, that is robust against time and wear [20]. The sources of undesirable variation, also called noises in this framework, are the following: 
- Variation in conditions of use

- Deterioration or variation with time and use

- Production or manufacturing variations.

These three types of noises cause degradation of performance or deviation away from ideal customer satisfaction. In this context, robustness is a characteristic of a system that minimizes these deviations, keeping performance economically close to ideal customer satisfaction [22]. The ideal quality a customer can receive is that every product delivers the target performance each time the product is used, under all intended operating conditions, and throughout its intended lifetime [20]. Put differently, robustness is a characteristic of a system whose performance is least sensitive to variations in operating environment, variation in raw material, thus allowing the use of low grade material and components, and variation in manufacturing, thus reducing labor and material cost for rework and scrap.

One of the goals of Robust Design is to exploit nonlinearities in the relation between a product quality characteristic and the various product parameters and noise factors in order to find a combination of product parameters values that gives the smallest variation in the value of the quality characteristic around the desired target value. This can be easily understood using the following mathematical formulation. Let $\mathbf{x}=\left(x_{1}, x_{2}, \ldots x_{n}\right)^{T}$ denote the noise factors and $\mathbf{z}=\left(z_{1}, z_{2}, \ldots z_{j}\right)^{T}$ the product parameters-called controlling factors-whose values can be set by the designer, then if the quality characteristic of the product is given by:

$$
y=f(\boldsymbol{x}, \boldsymbol{z})
$$

The deviation $\Delta y$ of the quality characteristic from the target value caused by small deviations $\Delta x_{i}$ of the noise factors from their nominal values can be approximated by the first terms of the Taylor series expansion of $f(\boldsymbol{x}, \boldsymbol{z})$ around $\boldsymbol{x}_{0}$ and $\boldsymbol{z}_{0}$ where $\boldsymbol{x}_{0}$ is the expected value of the noise factors and $z_{0}$ the unknown nominal settings of the product parameters:

$$
\Delta y \cong\left(\frac{\partial f}{\partial x_{1}}\right) \Delta x_{1}+\left(\frac{\partial f}{\partial x_{2}}\right) \Delta x_{2}+\ldots+\left(\frac{\partial f}{\partial x_{n}}\right) \Delta x_{n}
$$

The partial derivatives are evaluated at $\boldsymbol{x}_{0}$ and $z_{0}$. The above notation for the sensitivity coefficient is shorthand for:

$$
\left(\frac{\partial f}{\partial x_{i}}\right) \equiv\left(\frac{\partial f}{\partial x_{i}}\right)_{x_{0}, z_{0}}
$$

If the deviations in the noise factors are uncorrelated, the variance of quality characteristic can be expressed in terms of the variances of the individual noise factors as follows:

$\sigma_{y}^{2}=\left(\frac{\partial f}{\partial x_{1}}\right)^{2} \sigma_{x_{1}}^{2}+\left(\frac{\partial f}{\partial x_{2}}\right)^{2} \sigma_{x_{2}}^{2}+\ldots+\left(\frac{\partial f}{\partial x_{n}}\right)^{2} \sigma_{x_{n}}^{2}$

Thus the variance of the quality characteristic is the sum of the products of the variances of the noise factors times the sensitivity coefficients. The sensitivity coefficients are themselves function of the control factors as expressed in Equation (3). As can be seen from (4), the variance of the quality characteristic can be minimized by either selecting the control factors $z_{0}$ such that the sensitivity coefficients are minimum, or by reducing the variances of some of the noise factors, typically the tolerances on system's components. The first action is referred to as parameter design; the second is called tolerance design. Figure 5 illustrates the difference between achieving robustness via parameter design versus tolerance design.

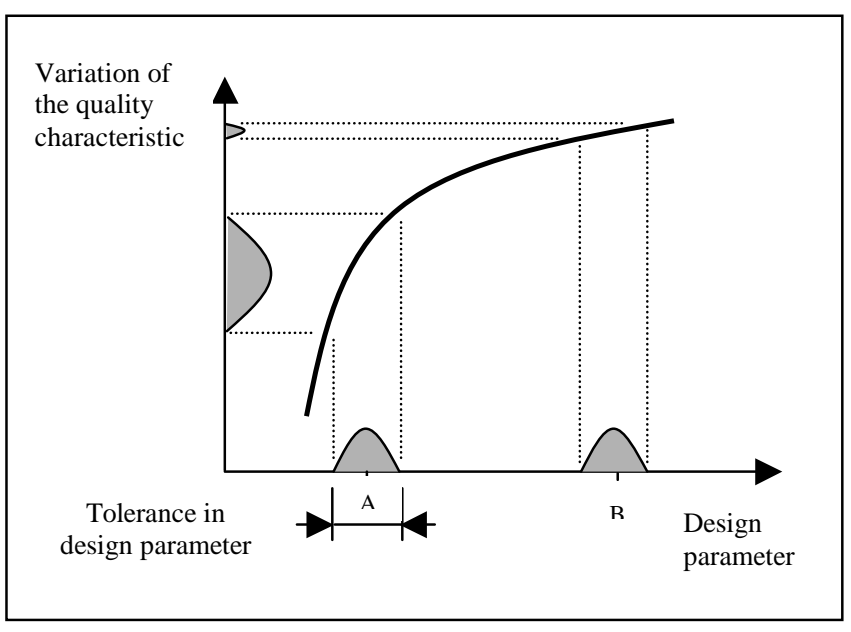

Figure 5. Variation of the quality characteristic as a function of the design parameter setting.

The above plot shows that a reduction in the variation of the quality characteristic of a product can be achieved by appropriately setting the design parameter, or control factor, at a point where the sensitivity-defined in Equation (3) - is small, e.g., choosing point B over point A. This is referred to as parameter design and does not 
affect the manufacturing cost of the component, as opposed to tolerance design that consists of reducing the tolerances on the design parameters, and is associated with more costly parts. From this perspective, it is clear that parameter design should be carried out prior to tolerance design in order to deliver robust products. This is a fundamental idea in Robust Design.

3.3.3. Synthesizing a Definition of Robustness. From the previous discussion, we can synthesize a general definition of robustness as the property of a system which allows it to satisfy a fixed set of requirements, despite changes occurring after the system has entered service, in the environment or within the system itself, from the nominal or expected environment or the system design parameters ${ }^{5}$.

For instance, in the case of Robust Design, the objective is to maintain a target performance despite the various noise factors such as the variations in the conditions of use of the system, the degradation of the system or system components with time, and the manufacturing variability. In the case of Robust Control, the fixed set of requirements is ensuring stability and maintaining some pre-defined performance specifications.

\subsection{Distinction Between Flexibility and Robustness of a Design}

The definitions discussed above provide a clear distinction between robustness and flexibility of a design. Although these two concepts refer to the ability of a system to handle change, the nature of the change, as well as the system's reaction to the change, in each case is very different: Flexibility, as defined herein, implies the ability of a design to satisfy changing requirements after the system has been fielded, whereas robustness involves satisfying a fixed set of requirements despite changes in the system's environment or within the system itself. The relation between flexibility and robustness of a design as a function of the system's objectives and environment is graphically illustrated in Figure 6.

\footnotetext{
${ }^{6}$ Design parameters are defined in [38] as the key physical variables that characterize a design and satisfy a set of specified requirements.
}

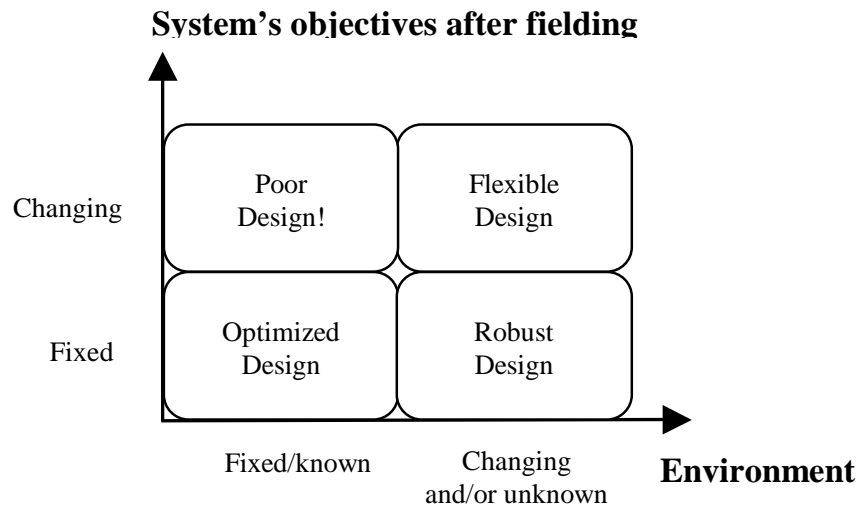

Figure 6. Flexibility and Robustness as a function of the system's objectives and environment.

The following thought experiment would help clarify the distinction between flexibility and robustness of a design. Imagine designing a spacecraft for 50-100 years! The two major challenges in striving for such a spacecraft design lifetime are the following:

1. Maintain on-board functionalities after launch, despite changes in software and hardware characteristics due to radiation impacts, malfunctions, aging, etc. This is indicative of the need for robustness of the design, i.e., robustness has to be built-in into the spacecraft.

2. Create new functionalities on-board for changes in requirements occurring after launch, as events unfold, new environments are explored, and/or new data becomes available, etc. Such changes are bound to happen given the extensive spacecraft design lifetime. This is indicative of the need for flexibility of the design, i.e., flexibility has to be embedded in the spacecraft.

\subsection{Distinction Between Universality and Flexibility of a Design}

Another distinction can be made, based on the definition of flexibility provided above, between two concepts that are potentially to be confused with one another: That of flexibility versus universality of a design. A software for instance that can be used in a variety of situations without change or modification, is considered "universal" not flexible. Flexible software [23] is one that can be easily changed-extended, contracted, or else-in order to be used in a variety of ways. Similarly, spacecraft that carry multiple instruments and performs multiple missions simultaneously are NOT considered flexible according to the definition of flexibility provided above. Likewise, a design is considered flexible if it is easily changeable to be used in a variety of ways. The time and 
cost required to implement the changes are two indicators of the "ease of change" of a design and reflect its flexibility.

\section{Examples: Flexibility and Product Design Lifetime}

The following examples illustrate the relationship between flexibility and design lifetime. The first example contrasts the operational lifetime of the Boeing B-52 with that of the Convair B-58, and makes the case that the B52 was a highly flexible design ${ }^{6}$. The second example discusses the need for flexibility in the rotorcraft industry, particularly in the light of the current restrictive military spending and the fact that helicopters are being designed for increasingly longer lifetimes. The third example argues that the Galileo spacecraft, by completing its initial objectives and performing a new or extended mission, constitutes an instance of flexibility in space systems.

\subsection{Designing for Flexibility: The Boeing B-52 versus the Convair B-58}

In order to illustrate the relationship between a product's life-span, the initial circumstances from which the system's requirements were derived and the various environments in which it can operate, the Boeing B-52 Stratofortress is presented as an example of a flexible design, and is contrasted with the Convair B-58 Hustler. The purpose of this section is not to delve into the particular design practices that enabled the B-52 to remain in operation long after the B-58 was retired, but simply to illustrate the above-mentioned relationship.

The B-52 is a long-range, heavy bomber that can perform a variety of missions. It is capable of flying at high subsonic speeds (Mach 0.86) at altitudes up to $50,000 \mathrm{ft}$, and carry both conventional and nuclear ordnance. In a conventional conflict, the B-52 can perform a variety of missions such as air interdiction, offensive counter-air, or maritime operations. It is capable of dropping or launching the widest array of weapons in the U.S. inventory including gravity bombs, cluster bombs, and guided missiles. The venerable aircraft has also been used to ferry both manned and unmanned systems for altitude drop and orbital insertion.

The B-52 first entered service in 1955 with the Strategic Air Command. The initial specifications were issued on November $23^{\text {rd }} 1945$. For the first 10 years of its Air Force service, it operated in a cold war atmosphere. Current engineering analysis shows the B-52 life-span can be extended beyond the year 2045. Thus it

\footnotetext{
${ }^{6}$ Although perhaps its flexibility was accidental.
}

will be a "century" aircraft. It has assumed important conventional roles in Vietnam and the Gulf war. These are very different environments from which the initial system requirements were derived-different environments thus different threats, hence the need to alter the tactics in order survive and prevail-No other weapons system offers the flexibility of the B-52. It is referred to as the bomber that "is not getting older, just getting better" because it was capable of accommodating numerous improvements over the years. Upgrades since the early 1980's have included many new and improved systems:

- Offensive avionics

- Environmental control

- Auto-pilot

- Enhanced electronic countermeasures

- Conventional air-launched cruise missile (CALCM)

The Convair B-58 Hustler on the other hand was the first supersonic bomber to enter service with the USAF in March 1960. Despite its high performance and sophisticated equipment, the service of the B-58 was brief; the aircraft flew for only a decade before being consigned to storage. Part of the reason for this rather short service was due to the aircraft's rather high accident rate. Another factor was the intercontinental ballistic missile, which entered service at the same time as the B58 and removed its primary mission. Of course the same was true of the B-52 but it proved flexible enough to find widespread use in other mission areas. Aside from the technical problems that plagued the B-58, the aircraft in some sense lacked the flexibility of the B-52 to adapt to new missions and roles in new environments.

It is tempting at this point to probe the original requirements of both the B-52 and B-58, and to identify the particular design choices that rendered on one hand the B-52 a flexible design to remain in operation for almost a century, and on the other hand the B-58 a shortlived inflexible design. The study should investigate for example the impact of the requirement to fly at supersonic speeds for the B-58 on the wing design and the airframe, and how this choice, later during the operational life of the B-58, prevented it from accommodating different weapons and performing other missions than the one the it was initially designed for. This is however beyond the scope of this section. The purpose of this example, as stated above, is to illustrate the relationship between flexibility and product life-span, and not to delve into the particular design choices that render a product flexible ${ }^{7}$.

\footnotetext{
${ }^{7}$ It is also possible that the complete story of the short-lived B-58 may not yet be known: The B-58 may have been consigned to storage for classified reasons.
} 


\subsection{The Need for Flexibility in the Rotorcraft Industry}

Helicopters tend to have an operational life-span exceeding 30 years. In many cases, this is long after the circumstances for the original requirements have been removed. Hence, the missions and roles of a rotorcraft are most likely to change over its life-span. Also, the embedded technologies within the rotorcraft continue to evolve after the product has been fielded. Due to the high value of these already fielded products, there is a tendency among the operators to modify the fielded rotorcraft to adapt to new missions and roles as opposed procuring new ones. Furthermore, the current state military spending in the United States forces traditional military contractors to seek non-traditional business segments of the market. Therefore fewer products have to be designed for an extended life-span and with the ability to perform new and diverse missions. Rotorcrafts in particular have to be designed with the ability to be modified after entering service in order to perform new and emergent missions. In other words, flexibility has to be embedded in the initial design.

Consider for instance the Sikorsky medium lift helicopter S-70 or its UH-60 designation for its military role. The helicopter was developed in the early 1970s in response to the U.S. Army rotary-winged aircraft program referred to as the Utility Tactical Transport Aircraft System (UTTAS). In short, the UTTAS program required a helicopter to perform multiple missions such as troop transport, air cavalry, and medical evacuation. The program also included standards for the helicopter's combat survivability, reliability, maintainability, as well as adverse weather and nighttime operational capabilities. Designed as a military utility helicopter, the UH-60/S-70 has now over 35 derivatives performing a variety of missions, e.g., troop transport, cargo movement, medical evacuation, VIP transport, and has been sold in over 90 countries [24]. The need to access new markets, or to satisfy specific customers requirements, led to the development of these derivatives. However, it is the intrinsic ability of the UH-60/S-70 baseline architecture to accommodate changes following new customers requirements-in a timely and cost-effective way in order to achieve a different configuration vehicle - that made it possible to develop these derivatives. Holmes (1999) argues that the use of platform design for medium lift helicopters enabled the expansion of the mission roles and capabilities of the UH-60/S-70 thus provided the flexibility of its baseline architecture.

\subsection{Galileo's Mission to Jupiter and the Galileo Europa Mission (GEM) Extension}

The Galileo spacecraft is a NASA robotic mission to explore Jupiter. The spacecraft consisted of an orbiter and an atmospheric entry probe designed to enter Jupiter's atmosphere and provide a weather report on temperature, pressure, composition, wind, and lightning of Jupiter's atmosphere. The spacecraft was launched on-board the Space Shuttle Atlantis in 1989 and reached Jupiter in 1995.

The initial science objectives of the Galileo orbiter included the following:

1a. Investigating the circulation and dynamics of the Jovian atmosphere and ionosphere

2a. Characterizing the vector magnetic field and the energy spectra, composition, and distribution of energetic particles and plasma to a distance of $150 \mathrm{R}_{\mathrm{J}}$

3a. Conducting long-term observation of its magnetosphere

4a. Characterizing the morphology, geology, and physical state of the Galilean satellites [Galileo Project Information] $]^{8}$

On December 1997, Galileo successfully completed its original mission objectives: A two-year study of the Jovian system. Since the resilient spacecraft was capable of much more, it was decided to extent the mission, now called the Galileo-Europa Mission (GEM), in order to study in detail Jupiter's icy moon Europa and its fiery moon Io. The new major science objectives of the GEM are the following:

1b. Europa: Study and characterize crust, atmosphere, and possible ocean (i.e., implication for exobiology) using imaging, gravity, and space physics data

2b. Io Plasma Taurus: Explore and map Io Plasma Taurus as orbit approaches Io.

3b. Io: Intensive study of Io's volcanic processes, atmosphere, and magnetosphere environment [GEM Fact Sheet, 00] ${ }^{9}$

The fact that the orbiter has completed its initial mission and performed a new or extended mission constitutes one instance of flexibility of a space system as defined above-ability to respond to changes in a system's initial objectives and requirements occurring after the system has been fielded. This flexibility was in part due to the various design margins that the orbiter had,

\footnotetext{
${ }^{8}$ Can be found at www.nssdc.gsfc.nasa.gov /galileo.html

${ }^{9} \mathrm{Can}$ be found at www.jpl.nasa.gov/galileo/gem/fact.html
} 
e.g., its design lifetime exceeded the time required to complete its science objectives $(\Delta \mathrm{V}$ margin, etc.). Other instances of flexibility in space systems are discussed below.

\section{Flexibility in the Context of Distributed Satellite Systems (DSS) and TechSat21}

Distributed space architectures, or the spreading of functionalities across multiple spacecraft, thus forming a virtual satellite, enable new missions to be performed, and often offer reduced cost or improved capabilities over monolithic designs ${ }^{10}$. Martin and Stallard (1999) discuss the application of DSS to synthesize a large space aperture:

"Since the satellites are not connected by structures, they can be separated over very large baselines that could not be considered for monolithic apertures. This feature can be beneficial for such missions as space-based radar, or large apertures for detection of slow moving targets in clutter. [...] Another mission application [of DSS] is mobile jam resistant communications [...] or interferometric imaging..."

The ability to reconfigure a cluster's geometry for instance allows modifying the revisit time requirement. This in one particular instance of flexibility-ability to respond to changes in the requirements occurring after the system has been fielded - that is characteristic of DSS and that is not feasible with a monolithic design. Furthermore, the ability to modify the revisit time (RT) on-orbit implies that it needn't be specified prior to launch or further upfront in the development phase of the system.

The idea that critical system requirements need not be narrowly specified prior to launch, because changes can be accommodated afterwards, is one particular advantage of the property of flexibility in design. It seems particularly important and valuable in defense oriented space systems for instance where the development times are of the order of 5 to 10 years, and changes are very likely to occur, as well as for systems that operate in uncertain environments.

TechSat21 is an Air Force Research Laboratory program designed to explore new technologies for lightweight and low-cost clusters of micro-satellites. One instance of flexibility of TechSat21 for example results from the ability to modify of the cluster geometry in order to operate in a Geo-location mode instead of the nominal Radar mode. This is illustrated in the Figure 7.

\footnotetext{
${ }^{10}$ An elaborate discussion distributed satellite systems is
} provided by Shaw (1999).

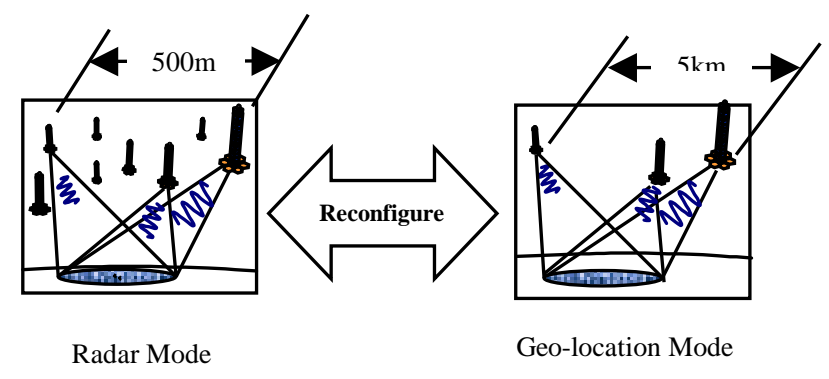

Figure 7. Reconfiguring the cluster geometry allows "other" missions to be performed. This is not feasible with a monolithic space system design.

Figure 8 illustrates two different types of flexibility associated with TechSat21: The first involves the ability of the system to change its mode of operation-in the parlance of the IEEE Standard 1233, this is relevant of the system's capability - whereas the second type involves the ability to modify the attribute of the requirement (tune-in performance).
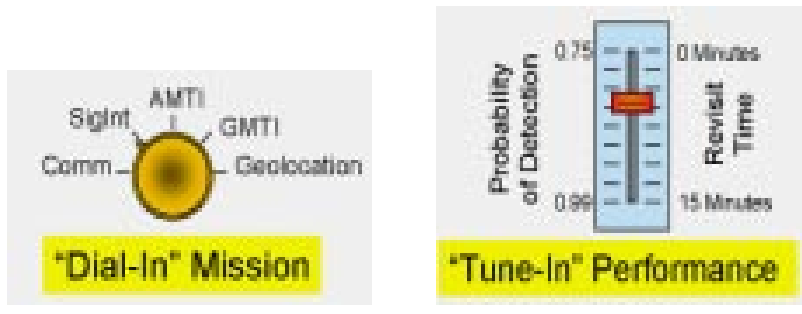

Figure 8. Two generic types of flexibility: The ability to modify the mode of operation of a system (Dial-In Mission), and the ability to modify the attribute of a requirement (Tune-In Performance).

\section{Summary and Conclusions}

This paper reviewed the concept of flexibility as discussed in various fields of investigations and extracted its characteristics features. Flexibility of a design is here defined as the property of a system that allows it to respond to changes in its initial objectives and requirements-both in terms of capabilities and attributes-occurring after the system has been fielded, i.e., is in operation, in a timely and cost-effective way.

In order to discuss any subject matter clearly, it is necessary to begin with a clear set of definitions. Indeed much can be gained through careful and consistent definitions of terms alone. Flexibility however has been a word rich with ambiguity. The first section of this paper identified the various sources of ambiguity in discussions of issues of flexibility: These include the failure of the 
familiar characterization of flexibility-ability to handle change-to distinguish it from other properties, particularly in the light of the proliferation of its pseudosynonyms. A selected literature review is then provided and a definition of flexibility is synthesized.

A brief literature review of Robust Control and robust Design is also presented. Robustness is defined as the property of a system that allows it to satisfy a fixed set of requirements, despite changes occurring after the system has entered service, in the environment or within the system itself, from the nominal or expected environment or the system design parameters.

Robustness and flexibility are then contrasted, and a distinction is drawn as well between flexibility and universality of a design. Flexibility of a design is also disentangled from issues of flexibility in the design process: The latter include activities, methods, and tools devised to mitigate the risks - cost, schedule, and performance-resulting from requirement changes occurring during the design process, i.e., before fielding a system.

Several examples of flexible systems are finally discussed, and illustrate the relationship between flexibility and a system's design lifetime. The examples included the (accidentally) flexible B-52-to remain in operation for almost a century-versus the short-lived inflexible B-58, the Galileo spacecraft; its initial mission to Jupiter and its extended mission to Io and Europa (the Galileo Europa Mission), as well as instances of flexibility in the context of distributed satellite systems.

Aside from the particular points referred to above, this paper also laid a framework for a clear and comprehensive discussion of issues of flexibility: One may disagree with the particular definitions provided herein, that's fair. The reader is encouraged to create his or her own set of definitions but should make sure that they are unambiguous, self-consistent, and lead to useful concepts. However a comprehensive treatment of flexibility in system design should address the following questions:

1. What is flexibility?

2. Why or when is flexibility needed in system design?

3. How can we design for flexibility? What are the design principles for embedding flexibility in system design?

4. What are the trade-offs associated with designing for flexibility? What is the value of flexibility and what are the penalties (cost, performance, risk, etc.), if any, associated with it?
This paper focused on the first question and extracted the characteristics features of flexibility. The following questions will be addressed in subsequent papers.

\section{References}

[1] R. McKinon. "The Concept of Flexibility and Urban Systems". Center of Urban Studies, University of Illinois, 1978.

[2] M. A. Fox, B. P. Yeh. "Intelligent Kinetic Systems in Architecture", in Managing Interactions in Smart Environments (MANSE). Springer-Verlag, London, 1999.

[3] M. Amram, N. Kulatilaka. Real Options: Managing Strategic Investments in an Uncertain World. Harvard Business School Press, 1999.

[4] A. Raouf, M. Ben-Daya (Editors). Flexible Manufacturing Systems: Recent Developments. Elsevier, 1995.

[5] J. A. Highsmith III. Adaptive Software Development: A Collaborative Approach To Managing Complex Systems. Dorset House Publishing, 1999.

[6] W. Chen, K. Lewis. "Robust Design Approach for Achieving Flexibility in Multidisciplinary Design". AIAA Journal, Vol. 37, No. 8, August 1999. pp. 982-989.

[7] L. Trigeorgis. Real Options: Managerial Flexibility and Strategy in Resource Allocation. MIT Press, 1996.

[8] F. Suarez, M. Cusumano, C. Fine. "Flexibility and Performance: A Literature Critique and Strategic Framework". Sloan School WP 3298-91-BPS, Massachusetts Institute of Technology, 1991

[9] T. Taylor. "Evaluating and Selecting Manufacturing Flexibility". S.M. thesis. Department of Mechanical Engineering, Massachusetts Institute of Technology, 1991.

[10] M. Piore. "Corporate Reform in American Manufacturing and the Challenge to Economic Reform". Mimeo, Massachusetts Institute of Technology, 1989

[11] General Accounting Office. "Best Practices: Better Matching of Needs and Resources will Lead to Better Weapon System Outcomes". GAO-01-288, March 2001. 
[12] A. Rapppaport Two-Person Game Theory. University of Michigan Press, 1969.

[13] Requirements Management Guidebook. SWELT: RM0.3-9/30/96.

[14] B. S. Blanchard. System Engineering and Management. Wiley-Interscience, 1998.

[15] J. Wertz, W. Larson (Editors). Space Mission Analysis and Design. Microcosm Press and Kluwer Academic Publishers, 1999.

[16] W. Chen, C. Yuan. "A Probabilistic-Based Design Model for Achieving Flexibility in Design". Journal of Mechanical Design, Vol.121, March 1999. pp. $77-83$.

[17] K. Lewis, F. Mistree. "Modeling Interactions in Multidisciplinary Design: A Game Theoretic Approach". AIAA Journal, Vol. 35, No. 8, June 1999, pp. 1387 - 1392.

[18] IEEE Guide for Developing System Requirements Specifications. 1998 Edition.

[19] Mars Global Surveyor: Spacecraft Requirements. JPL D-11509, 1996.

[20] M. S. Phadke. Quality Engineering Using Robust Design. Prentice-Hall, 1989.

[21] G. Taguchi. Systems of Experimental Design (English translation, edited by D. Clausing). UNIPUB/Kraus International Publications, New York, 1987.

[22] D. Clausing. Total Quality Development: A Step-byStep Guide to World-Class Concurrent Engineering. ASME Press, New York, 1994.

[23] D. Parnas. "Designing software for Ease of Extension and Contraction". IEEE Transactions on Software Engineering, Volume SE-5, Number 2, March 1979. pp. 128-138.

[24] C.Holmes. "Effective Platform Designs for Medium Lift Helicopters". S.M. thesis. Systems Design and Management, Massachusetts Institute of Technology, 1999.

[25] J. Ackerman. Robust Control: Systems with Uncertain Physical Parameters. Springer-Verlag, 1993.
[26] J. C. Doyle, B. Francis, A. Tannenbaum. Feedback Control Theory. Maxwell/Macmillan, 1992.

[27] M. A. Dahleh, I. J. Diaz-Bobillo. Control of Uncertain Systems: A Linear Programming Approach. Prentice-Hall, Englewood Cliffs, NJ, 1995.

[28] T. Faulkner. "Applying Options Thinking to R\&D Valuation”. Research-Technology Management, May-June 1996, 39(3).

[29] B. Francis. A Course in $H_{\infty}$ Control Theory. Springer-Verlag, Berlin, 1987.

[30] E. Fricke, A. Schulz, S. Wenzel, H. Negele. Design for Changeability of Integrated Systems within a Hyper-Competitive Environment, in INCOSE Colorado 2000 Conference, Denver, March 2000

[31] G. A. Hazelrigg. Systems Engineering: An Approach to Information-Based Design. Prentice-Hall, 1996.

[32] G. A. Hazelrigg. "An Axiomatic Framework for Engineering Design". Journal of Mechanical Design, Vol. 121, September 1999.

[33] M. Martin and M. Stallard. "Distributed Satellite Missions and Technologies: The TechSat21 Program”. AIAA-99-4479.

[34] S. U. Mohandas, E. Sandgren. "Multiobjective Optimization Dealing With Uncertainty". Advances in Design Automation, Vol. 19-2: 1989. pp. 241 248.

[35] J. Oleson. Pathways to Agility. Wiley, 1998.

[36] R. Sanchez-Pena, M. Sznaier. Robust Systems: Theory and Applications. Wiley, 1998.

[37] G. B Shaw. "The Generalized Information Network Analysis Methodology for Distributed Satellite Systems". PhD thesis, Department of Aeronautics and Astronautics, Massachusetts Institute of Technology, February 1999.

[38] N. P. Suh. Principles of Design. Oxford University Press, 1990.

[39] N. P. Suh. Axiomatic Design: Advances and Applications. Oxford University Press. (to be published). Manuscript format, 1999. 
[40] R. T. Stefani, C. J. Savant, B. Shahian, G. H. Hostetter. Design of Feedback Control Systems. Saunders College Publishing, 1994.

[41] D. L. Thurston. "A Formal Method for Subjective Design Evaluation with Multiple Attributes". Research in Engineering Design, Vol. 3: 1991. pp. $105-122$.

[42] A. Triantis, J. Hodder. "Valuing Managerial Flexibility". The Journal of Finance, Vol. XLV, No.2. June 1990.
[43] L. Trigeorgis, J. Mason in "Valuing Managerial Flexibility". Midland Corporate Finance Journal, 51987. pp. 14-21.

[44] D. R. Wallace, M. Jakiela, W. C. Flowers. "Design Search Under Probabilistic Specifications Using Genetic Algorithms". Computer-Aided Design, Vol. 28, N0. 5, 1996. pp. $404-406$.

[45] NASA Systems Engineering Handbook, SP-6105, June 1995. 